\title{
Retrieving wind statistics from average spectrum of continuous-wave lidar
}

\author{
E. Branlard ${ }^{1}$, A. T. Pedersen ${ }^{1}$, J. Mann ${ }^{1}$, N. Angelou ${ }^{1}$, A. Fischer ${ }^{1}$, T. Mikkelsen ${ }^{1}$, M. Harris ${ }^{2}$, C. Slinger ${ }^{2}$, and \\ B. F. Montes ${ }^{3}$ \\ ${ }^{1}$ DTU Wind Energy, Ris $\varnothing$ Campus, Technical University of Denmark, Roskilde, Denmark \\ ${ }^{2}$ ZephIR Ltd., The Old Barns, Fairoaks Farm, Hollybush, Ledbury, HR8 1EU, UK \\ ${ }^{3}$ LM Wind Power, Lunderskov, Denmark
}

Correspondence to: E. Branlard (ebra@dtu.dk)

Received: 5 November 2012 - Published in Atmos. Meas. Tech. Discuss.: 19 February 2013

Revised: 17 May 2013 - Accepted: 6 June 2013 - Published: 15 July 2013

\begin{abstract}
The aim of this study is to experimentally demonstrate that the time-average Doppler spectrum of a continuous-wave (cw) lidar is proportional to the probability density function of the line-of-sight velocities. This would open the possibility of using $\mathrm{cw}$ lidars for the determination of the second-order atmospheric turbulence statistics. An atmospheric field campaign and a wind tunnel experiment are carried out to show that the use of an average Doppler spectrum instead of a time series of velocities determined from individual Doppler spectra significantly reduces the differences with the standard deviation measured using ordinary anemometers, such as ultra-sonic anemometers or hotwires. The proposed method essentially removes the spatial averaging effect intrinsic to the cw lidar systems.
\end{abstract}

\section{Introduction}

Doppler lidars cannot precisely estimate the wind velocity fluctuation statistics in comparison to ordinary sonic anemometers because of the relatively large averaging volume. Many engineering applications such as the load estimation on buildings, and in particular loads on wind turbines, use point-wise measurements to characterise the turbulence at the site of the structure. To be consistent with the building and the wind turbine codes, it is desirable to have the lidars compensate for the averaging effect.

Many authors devise methods to measure turbulence statistics such as spectra, variances or the energy dissipation. The methods for estimating the energy dissipation (Frehlich and Cornman, 2002; O’Connor et al., 2010) typically assume a spectral shape, e.g. the Kolmogorov or von Kármán spectrum, and combine that with detailed knowledge about the line-of-sight (LOS) weighting function to derive this small-scale turbulence quantity. Similarly the effects of averaging over the measurement volume are sometimes estimated using complicated turbulence models. This is done for both single-beam systems (Drobinski et al., 2000; Lothon et al., 2009; Sjöholm et al., 2009) and multiple-beam systems (Mann et al., 2009). For the variances, Sathe et al. (2011) investigated the effect of averaging for conically scanning Doppler lidars. These lidars, which included both pulsed and continuous-wave (cw) systems, provided the three components of the velocity every few seconds by combining the LOS velocities obtained from the scans, and they are used widely by the wind energy industry (Smith et al., 2006; Emeis et al., 2007). Sathe et al. (2011) showed that the spatial averaging and the way LOS velocities are combined had a dramatic influence on the standard deviation of the velocity components, while Sathe and Mann (2012a) showed similar influences on the measured turbulence spectra. The vertical component variances are highly underestimated by the lidars, while the variances of the horizontal components are underestimated under stable conditions and overestimated under unstable conditions. The amount of under- or overestimation depends on the height in different ways for the two systems. Pichugina et al. (2008) showed that the calculation of the horizontal velocity variance depended on various parameters controlling both the temporal and spatial averaging in a vertical-slice scanning pulsed lidar. In all of the 
above investigations, the Doppler velocity is derived from the Doppler spectrum and then a statistical analysis of these Doppler velocities is carried out. In the present contribution, we average the Doppler spectra directly without calculating the Doppler velocities first. Following this approach, Mann et al. (2010) devised a method to circumvent the averaging effect completely for the vertical flux of the horizontal momentum for a conically scanning cw lidar. The method was based on the assumption that the Doppler spectrum averaging over an extended time period is equal to the probability density function (pdf) of the weighted LOS velocities in the measurement volume. The velocities are adjusted with a certain weighting, which for an untruncated, focused Gaussian beam is well approximated by a Lorentzian function (Sonnenschein and Horrigan, 1971).

The purpose of the present study is to experimentally verify the assumption that the average Doppler spectrum of a cw lidar is proportional to the pdf of the LOS velocities in order to promote the possibility of using lidars for precisely estimating the second-order turbulence statistics.

A field measurement campaign was carried out at Ris $\varnothing$ where a ZephIR prototype (Smith et al., 2006) was mounted on a parked Vestas V27 turbine nacelle staring horizontally upwind towards a mast-mounted sonic anemometer (Angelou et al., 2012b).

A wind tunnel experiment was performed in order to investigate what other effects other than turbulence might contribute to the width of the mean Doppler spectrum. Further, in the wind tunnel, by adding a grid, we generated turbulence with a small length scale of the order of a few centimetres. Under these conditions the averaging effect of the lidar was considerably larger than in the field experiment and the advantage of deriving turbulence statistics from the average Doppler spectrum was more evident.

Following the work of Mann et al. (2010) the method and assumptions used for determining the wind statistics from the Doppler spectra are presented in Sect. 2 along with considerations about possible additional spectral broadening. Some practical aspects of the manipulation of raw Doppler spectra are discussed in Sect. 3. In Sects. 4 and 5, the field and wind tunnel experiments are described, followed by a presentation of the different results and discussions.

\section{Lidar Doppler spectra for wind analysis}

Commercial Doppler lidars are commonly used as anemometers in which the velocity is directly calculated by the internal software provided by the lidar's manufacturer. This velocity is obtained from the Doppler spectrum by various estimation techniques. The use of Doppler spectra for accessing the statistics of the LOS velocity at the focus point of the lidar will be discussed. Under some assumptions, it will be seen that the spatial averaging of the lidar can be circumvented.

\subsection{Lidar Doppler spectra}

The LOS is oriented by a unit vector $\boldsymbol{n}$ in the direction of emission. The beam waist or focus point is located at a position $\boldsymbol{x}$ in space corresponding to a radial position $s=0$ along the LOS. The radial velocity (or LOS velocity) measured by the lidar is assumed to be a weighted average of all the velocities along the LOS; therefore,

$v_{\mathrm{r}}(\boldsymbol{x})=\int_{-\infty}^{\infty} \varphi(s) \boldsymbol{n} \cdot \boldsymbol{u}(s \boldsymbol{n}+\boldsymbol{x}) \mathrm{d} s$,

where $\varphi$ is the lidar weighting function and $\boldsymbol{u}$ is the threedimensional velocity field. For the ZephiR lidar, and for any well constructed cw lidar, this function is well approximated by a Lorentzian (Smith et al., 2006; Angelou et al., 2012b). The lower limit of the integration should rigorously correspond to the position of the lidar. It is taken here as $-\infty$ for simplicity. This choice is also convenient since the Lorentzian function does not have a compact support. Nevertheless, this limit could be replaced by the position of the lidar if it is ensured that the weighting function is normalised to unity on the integration domain. This is the property of the weighting function used in Sect. 2.2.

In practice, the lidar measures the frequency difference $\Delta f$ between the emitted beam and the back-scattered radiation from atmospheric aerosols. To assess this frequency difference, the beat signal between the local oscillator and the backscattered radiation is Fourier transformed and absolute squared in order to obtain a spectrum denoted as $S(f)$. The determination of the centre frequency is not completely straightforward as discussed in Sect. 3.1. The frequency shift assessed into the spectral domain is converted to the wind radial velocity $v_{\mathrm{r}}(x)$ using

$\frac{\Delta f}{f}=2 \frac{v_{\mathrm{r}}}{c} \Rightarrow v_{\mathrm{r}}=\frac{1}{2} \lambda \Delta f$,

with $f, \lambda$ and $c$ being the lidar frequency, lidar wavelength, and speed of light, respectively. Using Eq. (2), we can express the Doppler spectrum in terms of velocity: $S\left(v_{\mathrm{r}}\right)$.

\subsection{Average lidar Doppler spectra}

In this section, the mathematical formalism related to the time averaging of the Doppler spectra is introduced.

Mann et al. (2010) made a simple assumption about the connection between spectrum and velocity distribution: the Doppler power spectral density corresponding to a given velocity $\tilde{v}_{\mathrm{r}}$ is taken as the sum of the contributions of all the radial velocities equal to $\tilde{v}_{\mathrm{r}}$ along the beam weighted by the spatial averaging function $\varphi$ :

$S\left(\tilde{v}_{\mathrm{r}}\right)=\int_{-\infty}^{\infty} \varphi(s) \delta\left(\tilde{v}_{\mathrm{r}}-v_{\mathrm{r}}(s)\right) \mathrm{d} s$. 
The tilde notation will be further dropped for simplicity. It should be noted that in the above equation, the spectrum is assumed to be normalised to unity for the sake of simplicity. The Doppler spectra are averaged for a given period, and this time averaging is denoted by $\langle\ldots\rangle$. The average spectrum is as follows:

$\left\langle S\left(v_{\mathrm{r}}\right)\right\rangle=\int_{-\infty}^{\infty} \varphi(s) p\left(v_{\mathrm{r}} \mid s\right) \mathrm{d} s$,

where $p\left(v_{\mathrm{r}} \mid s\right)$ is the probability density function (pdf) of the radial velocities at a radial position $s$ on the beam during the time period investigated.

Mann et al. (2010) pointed out that if the pdf of the velocities does not depend on the position $s$, then $p\left(v_{\mathrm{r}} \mid s\right)=p\left(v_{\mathrm{r}}\right)$ and Eq. (4) becomes:

$\left\langle S\left(v_{\mathrm{r}}\right)\right\rangle=p\left(v_{\mathrm{r}}\right)$.

In the two experiments considered in this paper, the beam direction is in a homogeneous direction, meaning that the statistics of the velocity does not change along the beam, and Eq. (5) is valid. In Mann et al. (2010), the mean wind speed changes along the beam; hence, if $p\left(v_{\mathrm{r}} \mid s\right)$ depends on $s$, Eq. (4) must be used instead of Eq. (5).

\subsection{Other sources of spectral broadening}

Scanning the lidar beam through the atmosphere or staring at an angle as compared to the direction of the wind flow will lead to spectral broadening of the signal even if the flow itself is perfectly homogeneous. This is essentially because the time a scattering particle (or aerosol) is illuminated by the lidar beam is limited. The bandwidth of the signal is inversely proportional to the time that an aerosol takes to move through the beam diameter. We perform a simplified analysis of how this affects the spectrum by assuming that the aerosol passes the beam close to the focus. We ignore the Doppler shift itself and focus on the width of the Doppler spectrum.

The cross section of the electric field in the focus point of the beam can be described by a centred Gaussian

$E(x) \propto \exp \left[-\left(\frac{x}{w_{0}}\right)^{2}\right]$,

where $w_{0}$ is the beam waist radius $\left(1 / e^{2}\right.$ half-width $)$ of the optical power, which is the square of the electrical field, and $x$ is the position perpendicular to the beam. The time scale for a scatterer moving perpendicular through the beam waist is

$\tau=\frac{w_{0}}{v_{\text {part }}}$,

where $v_{\text {part }}$ is the relative velocity between the focus point and the particle. The voltage, generated by the detector, will also be a Gaussian as a function of time

$V(t) \propto \exp \left[-\left(\frac{t-t_{0}}{\tau}\right)^{2}\right]$,

where $t_{0}$ is the random timing of the aerosol transit. In the frequency domain, the detector output becomes

$V(f) \propto \exp \left[-(\pi \tau f)^{2}\right] \exp \left[2 \pi \mathrm{i} f t_{0}\right]$,

and hence, the power spectrum $S(f)$, which is the mean absolute square of the above, becomes proportional to $\exp \left[-2(\pi \tau f)^{2}\right]$. Therefore, the $1 / e^{2}$ full-width, or the socalled spectral bandwidth, is

$f_{\mathrm{BW}}=\frac{2}{\pi \tau}=\frac{2 v_{\text {part }}}{\pi w_{0}}$.

An additional effect that widens the Doppler spectrum is the finite time $T$ used as a window in the Fourier analysis of the detector signal. Now, the detector signal becomes

$V(t) \propto \exp \left[-\left(\frac{t-t_{0}}{\tau}\right)^{2}\right] \Pi(t / T)$,

where $\Pi(t / T)$ is the rectangle function, i.e. $1 / T$ for $t \in$ $[-T / 2, T / 2]$ and zero otherwise. The Fourier transform of Eq. (11) is

$$
\begin{aligned}
V(f) & \propto \int_{-T / 2}^{T / 2} \exp \left[-\left(\frac{t-t_{0}}{\tau}\right)^{2}\right] \exp [-2 \pi i f t] \mathrm{d} t \\
& \propto \exp \left[2 \pi \mathrm{i} f t_{0}\right] \exp \left[-(\pi f \tau)^{2}\right] \\
\times & {\left[\operatorname{erf}\left(\frac{1}{2} \frac{T}{\tau}-\frac{t_{0}}{\tau}-\mathrm{i} \pi f \tau\right)-\operatorname{erf}\left(\frac{1}{2} \frac{T}{\tau}+\frac{t_{0}}{\tau}+\mathrm{i} \pi f \tau\right)\right], }
\end{aligned}
$$

where erf is the error function of a complex argument. Taking the mean square of this equation implies averaging over the random time $t_{0}$, so

$$
\begin{aligned}
& S(f) \propto \exp \left[-2(\pi f \tau)^{2}\right] \times \int_{-\infty}^{\infty} \mid \operatorname{erf}\left(\frac{1}{2} \frac{T}{\tau}-\frac{t_{0}}{\tau}-\mathrm{i} \pi f \tau\right) \\
& +\left.\operatorname{erf}\left(\frac{1}{2} \frac{T}{\tau}+\frac{t_{0}}{\tau}+\mathrm{i} \pi f \tau\right)\right|^{2} \mathrm{~d} t_{0},
\end{aligned}
$$

where the integral has to be calculated numerically. The resulting spectral shapes approach a Gaussian for $T \gg \tau$ and a $\operatorname{sinc}^{2}$ for $T \ll \tau$. In Sect. 6.5 the widths are computed by fitting a Gaussian to Eq. (13) to obtain the $1 / e^{2}$ full-width using the parameters of the wind tunnel experiment $\left(w_{0}=57 \mu \mathrm{m}\right.$, and using Eq. (7) to get $\tau$ and $T=5 \mu \mathrm{m}$ ). The sinc ${ }^{2}$ function is not fitted well with a Gaussian, but is similar to the way widths are obtained from the data. Parallel considerations may be found in Kristensen et al. (2011). 
The derivation presented here is only approximate. The purpose of the derivation is to show the proportionality under non-turbulent flow conditions of the Doppler spectral width with the wind speed as expressed in Eq. (10), and the small additional offset due to the Fourier analysis time $T$.

\section{Problems raised by the manipulation of raw spectra}

\subsection{Determination of the frequency}

Determining the radial velocity $v_{\mathrm{r}}$ from the lidar Doppler spectrum is in reality more complex than stated in Eq. (2). The averaging of the Doppler spectra allows a better signalto-noise ratio, and this first averaging step is carried out internally by the lidar. Once the signal is sufficiently significant, the challenge lies in the determination of the main frequency of the spectrum. The different techniques employed by the manufacturers are beyond the scope of this paper. Nevertheless, a method to determine the main frequency of the spectrum had to be used for this study and a choice had to be made between three different methods. First, the frequency can be considered to correspond to the maximum power spectral density. Secondly, the frequency can be considered the barycenter of the power density. Finally, the frequency can be determined as the median of the spectrum. This last method has been used in the analysis of the lidar spectra presented here to determine the lidar wind speed time series (also see Angelou et al., 2012a). It should be noted that the analytical development of Sect. 2.1 and Eq. (1) use the barycenter definition. The barycenter definition is easier to handle analytically, but the median method is seen to be less noisy in practice (Angelou et al., 2012a).

\subsection{Different scaling for the lidar spectra}

Post processing of the raw ZephIR lidar spectra is required prior to the application of the frequency estimator method used to derive the Doppler shift. The first step is to divide the spectrum with the background noise spectrum in order to obtain a flat background. Then, the background noise of the spectrum is removed, and for convenient storage, the signals are scaled so that they take values in the same range. These two steps are performed as follows:

Each frequency spectrum $S(f)$ output by the lidar is scaled and stored in a database according to

$S_{\mathrm{DB}}(f)=255 \times \frac{S(f)-S_{\text {noise }}}{S_{\max }-S_{\text {noise }}}=\alpha S(f)+\beta$.

The noise threshold level $S_{\text {noise }}$ is determined by an interval away from the frequency corresponding to the maximum of the spectrum $S_{\max }$. The Doppler shift signal is indeed expected to be where the spectrum is maximum so that far from this maximum, the spectrum consists of the flat background noise. The noise threshold is given by the mean plus, somewhat arbitrary, three times the standard deviation of the spectrum on this interval (Angelou et al., 2012a). The spectra stored in the database $S_{\mathrm{DB}}$ take values between 0 and 255 .

At this stage, all database spectra have the same amplitude for a convenient storage, but prior to averaging them over ten or thirty minutes, the spectra are scaled a second time by division with another scaling factor $\alpha^{*}$. For this second stage three different scaling methods are considered. The first possibility is to keep the database spectra as they are $\left(\alpha^{*}=1\right)$ implying the same contribution to the average spectrum from signals with low backscatter and signals with high backscatter. Another possibility is to scale back the database spectra to get to the original lidar spectra without noise $\left(\alpha^{*}=\alpha\right)$. Finally, the third option involves normalising each spectrum by its area $\left(\alpha^{*}=\int S_{\mathrm{DB}}(f) \mathrm{d} f\right)$ assuming a constant energy content for all the spectra. After scaling, the frequency spectra are converted to velocity spectra according to Eq. (2), then averaged over a given period, typically $30 \mathrm{~min}$, and normalised to have unit area. All methods were tested on the field experiment described in Sect. 4, and as later described the last method was slightly superior, and was applied for the wind tunnel experiment in Sect. 5.

\section{Field experiment}

The field experiment used to validate Eq. (5) and show the benefit of analysing average Doppler spectra for turbulence statistics will be described in this section.

From April 2009 to December 2009 a measurement campaign was carried out at the DTU Ris $\varnothing$ campus test site where a ZephIR prototype lidar was mounted on the nacelle of a Vestas V27 wind turbine staring horizontally. During one day, the turbine was stopped and its yaw locked so that the laser beam focused $1 \mathrm{~m}$ above a Metek USA sonic anemometer measuring at $32 \mathrm{~Hz}$ and located on a meteorological mast $67.5 \mathrm{~m}$ from the lidar. The two instruments were covering exactly the same time interval and the measurement volumes were almost co-located which facilitates the comparison of turbulence statistics between the instruments. The Metek sonic was corrected offline according to the wind-tunnel-based calibration provided by the manufacturer (Berg et al., 2011). The sampling rate of the lidar is $100 \mathrm{MHz}$ which leads to a velocity resolution of roughly $0.15 \mathrm{~m} \mathrm{~s}^{-1}$ using $512 \mathrm{pt} \mathrm{FFT.} \mathrm{Refer} \mathrm{to} \mathrm{Angelou} \mathrm{et} \mathrm{al.} \mathrm{(2012b)}$ for further details.

To account for possible installation misalignment, the direction of the lidar's line-of-sight was determined by performing a correlation between the sonic wind speed projected on different angles and the lidar radial velocity using 10-min statistics. The time series from the lidar and the sonic anemometer were synchronised using the lidar time series as a reference and determining the time shift by crosscorrelation. Periods where the radial velocity was below $4 \mathrm{~m} \mathrm{~s}^{-1}$ were ignored. Indeed, the precision of the lidar drops at low wind speeds because relative intensity noise (RIN) 
from the fibre laser and centred at around $1 \mathrm{MHz}$ dominates the lidar spectrum at low frequencies (Cariou et al., 2006). This leads to a decrease in signal-to-noise ratio and makes it more difficult to assess the Doppler shift. The effect of RIN can be cancelled by using balanced photo detectors in the lidar, or possibly a laser with active RIN suppression (Spiegelberg et al., 2004). Excess RIN has been eliminated from the current generation of ZephIR lidars, which operate close to the shot noise limit across the full speed range. The threshold of $4 \mathrm{~m} \mathrm{~s}^{-1}$ was chosen to match the usual value of wind turbines cut-in wind speed.

In this experiment, the lidar stared horizontally over a terrain that had a slight slope of the order of $2 \%$. As the first approximation it was assumed that the flow was homogeneous along the LOS. The relation $p\left(v_{\mathrm{r}} \mid s\right)=p\left(v_{\mathrm{r}}\right)$ was hence assumed, and data from this experiment were used for validating Eq. (5).

\section{Wind tunnel experiments}

Two experiments, one with and the other without turbulence, were carried out in a wind tunnel.

\subsection{Turbulence}

To verify the expression given in Eq. (5), a comparison study between a hotwire anemometer (HW) and a shortrange $\mathrm{cw}$ lidar was performed in an aerodynamic wind tunnel. The wind tunnel had a test section with the dimensions of $7 \times 2.7 \times 1.35 \mathrm{~m}^{3}$ and was capable of delivering wind speeds up to $100 \mathrm{~m} \mathrm{~s}^{-1}$ (Fischer, 2012). To create a turbulent airflow, a metal grid was placed in the inlet of the test section. The HW was a 2-D X-wire probe (Dantec 55P61), and it was placed in the middle of the wind tunnel test section measuring two perpendicular components of the wind velocity, one along the wind and the other in the transverse direction, at a rate of $25 \mathrm{kHz}$ (Fischer, 2012). The lidar was a ZephIR $300 \mathrm{cw}$ lidar modified to measure high wind speeds of up to $80 \mathrm{~m} \mathrm{~s}^{-1}$. The sampling frequency and velocity resolution of the lidars used for the field and wind tunnel experiment are identical. However, the most important modification was the replacement of the standard telescope unit with a smaller and externally connected telescope. This telescope had a 1inch $(2.54 \mathrm{~cm})$ lens that gave the lidar a measurement range of approximately $1-10 \mathrm{~m}$ and was connected to the laser and signal processing unit through a $35 \mathrm{~m}$ optical fibre duplex cable such that it could be mounted inside the wind tunnel with a bulky base unit placed outside (Pedersen et al., 2012). The lidar stared horizontally into the wind with the waist of the laser beam placed approximately $2 \mathrm{~cm}$ above the HW.

Two series of tests were conducted in which the tunnel was set to deliver a mean wind speed of 45 and $55 \mathrm{~m} \mathrm{~s}^{-1}$, respectively. The hotwire recorded data for $83.9 \mathrm{~s}$ at a rate of $25 \mathrm{kHz}$ while the lidar sampled for $60 \mathrm{~s}$ at $50 \mathrm{~Hz}$. From the
HW data, the velocity component in the staring direction of the lidar was calculated, and from this, the pdf was subsequently calculated by dividing the range from $41-47 \mathrm{~m} \mathrm{~s}^{-1}$ (or 51-57 $\mathrm{m} \mathrm{s}^{-1}$ ) into 100 bins and counting the occurrences. For the lidar, two different approaches were used. In the first approach, the ensemble averaged mean spectrum was calculated as described in Sect. 2.2. In the second approach, one wind speed is calculated from each $50 \mathrm{~Hz}$ Doppler spectrum using the median method, and then, the pdf was found as for the HW. It should be pointed out that the determination of the median was not restricted to bin values, intermediate values using linear interpolation were allowed.

\subsection{Doppler spectral width in laminar flow}

To experimentally examine the spectral broadening due to reduced dwell time, the lidar was placed in the wind tunnel without the grid such that a laminar flow was achieved. This time a telescope with a 2-inch $(5.08 \mathrm{~cm})$ lens was used, and the lidar beam was focused at a distance of $1.05 \mathrm{~m}$, well inside the boundaries of the test section, and pointed downwards at an angle of $66.5^{\circ}$ as compared to the horizontal wind flow. The short focus range resulted in a tight focus with a waist radius of $52 \mu \mathrm{m}$, but because of the angle between the direction of the beam and the flow, the effective waist radius was $57 \mu \mathrm{m}$. Using this value for the waist radius, we could rewrite Eq. (10) as

$f_{\mathrm{BW}}=11163 \mathrm{~m}^{-1} \cdot v_{\text {part }}$.

The wind speed was ramped up from 10 to $70 \mathrm{~m} \mathrm{~s}^{-1}$ in steps of $10 \mathrm{~m} \mathrm{~s}^{-1}$, and the data were collected for two minutes in each step. The lidar sampled 50 spectra per second, and after spectra not containing a wind signal were discarded, approximately 5820 usable spectra were obtained for each step. The spectra were then ensemble averaged as described in Sect. 3.2 and subsequently a Gaussian distribution was fitted by a least-squares technique to obtain the width.

\section{Results}

In the field experiment described in Sect. 4, the pdf of the radial velocities at the measurement point can be assessed in three different ways using the data from the lidar and the sonic anemometer. The reference pdf is the one obtained from the wind speed time series of the sonic anemometer projected on the LOS of the lidar. From the wind speed time series of the lidar that corresponds to the weighted average of the radial wind speeds along the beam (according to Eq. 1), another pdf is derived. Eventually, provided the assumptions that led to Eq. (5), averaging the Doppler spectra from the lidar during a given period should give an unbiased assessment of the pdf of the radial velocities at the focus point for this period. Here, the focus is to prove the validity of Eq. (5) by the means of the experimental setup on the V27 nacelle at the DTU Ris $\varnothing$ campus. 


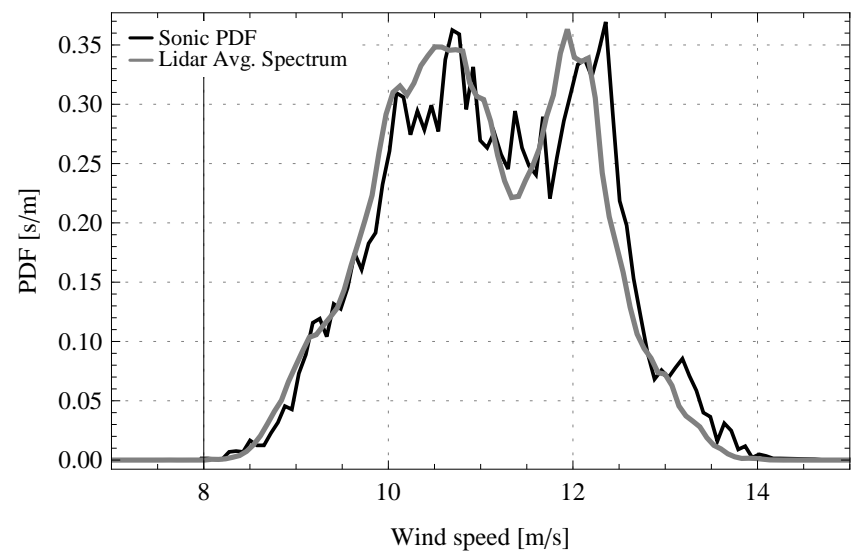

Fig. 1. Comparison of sonic pdf with the corresponding lidar average spectrum for a period of $30 \mathrm{~min}$.

An example of the comparison between the sonic pdf for a given period and the corresponding lidar spectrum is shown in Fig. 1. As can be seen, the curves correlate to a high degree but a small shift is observed. This shift is found from the small difference in the mean wind speed measured by the two instruments. This will be further investigated in the following paragraphs.

\subsection{Comparisons of statistical moments}

The mean is derived from the pdf as

$\mu_{v_{\mathrm{r}}}=\int_{-\infty}^{\infty} v_{\mathrm{r}} p\left(v_{\mathrm{r}}\right) \mathrm{d} v_{\mathrm{r}}$,

and the standard deviation as

$\sigma_{v_{\mathrm{r}}}^{2}=\int_{-\infty}^{\infty}\left(v_{\mathrm{r}}-\mu_{v_{\mathrm{r}}}\right)^{2} p\left(v_{\mathrm{r}}\right) \mathrm{d} v_{\mathrm{r}}$.

Using the wind speed time series from the lidar and the sonic anemometer, we find that these moments correspond exactly to the moment obtained by using ensemble averaging over the studied period, which is $\mu_{v_{\mathrm{r}}}=\left\langle v_{\mathrm{r}}\right\rangle$ and $\sigma_{v_{\mathrm{r}}}^{2}=\left\langle v_{\mathrm{r}}^{2}\right\rangle-\left\langle v_{\mathrm{r}}\right\rangle^{2}$. The comparison of the mean radial velocity from the lidar and the sonic shows a really high correlation with a slope of 0.993 as shown on Fig. 2. A slightly inferior slope of 0.992 is found by comparing the mean from the average spectrum calculated with Eq. (16) and the time series of the sonic anemometer. As seen in Fig. 1, this small shift in the mean wind speed is visible when comparing the two pdfs. As a result, the small difference in mean is corrected for further comparisons of the pdfs. The difference in mean is discussed in Sect. 7. The results for the field experiment concerning the standard deviation will be presented in Sect. 6.3.

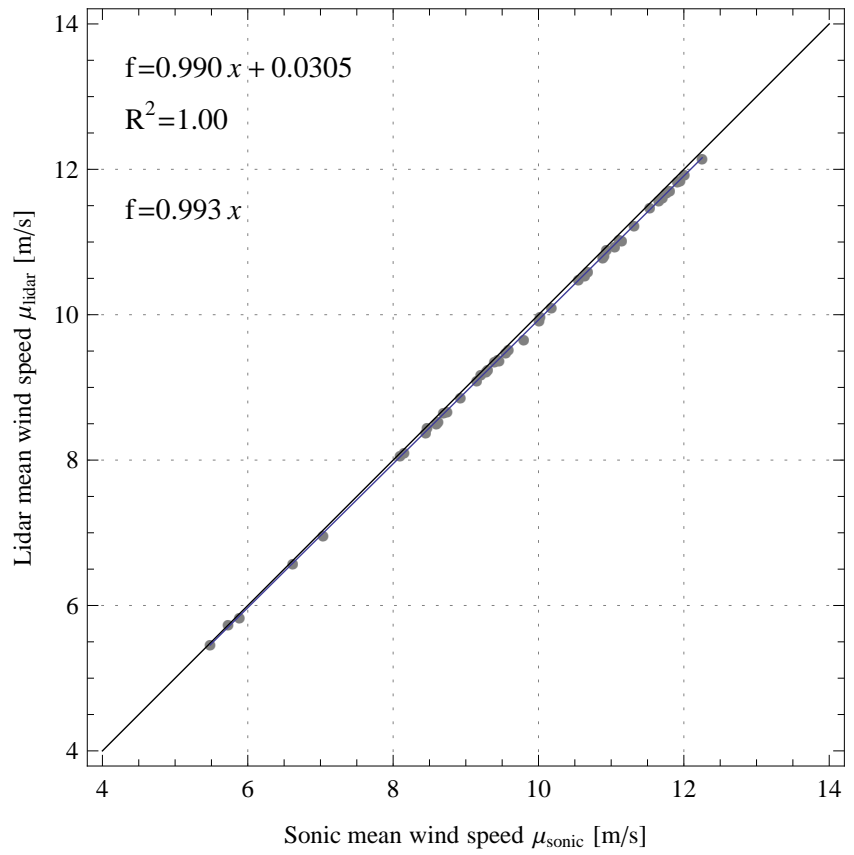

Fig. 2. Comparison of half-hour averages of the LOS velocity from the lidar and the corresponding velocity component of the sonic. Expressions for a linear fit with and without an offset are shown. Data are obtained during the day of 5 May 2009.

\subsection{Comparison of sonic pdf with average lidar spectra}

Averaging periods from $30 \mathrm{~s}$ to $30 \mathrm{~min}$ have been investigated for comparisons with the sonic pdf. Results for $10 \mathrm{~min}$ or 30 min periods are presented in the following paragraphs as they are reasonable periods for evaluating turbulence statistics and their resulting pdf is relatively smooth. A correlation method is presented to quantify the similarity between the sonic pdf and the average Doppler spectrum. As the first means of comparison, the sonic pdf and the average spectrum are plotted above each other as shown in Fig. 3. This instationary case corresponds to a $30 \mathrm{~min}$ period where the radial velocity decreased significantly, thus explaining the observed double spikes. The correction for the small difference in mean is taken into account for these plots and it can be seen that the curves agreement is remarkable. Such graphical comparisons were performed for 1330 min periods and 46 10 min periods, showing similar results.

To quantify the similarities between the shapes of the pdfs, a correlation between the curves is performed with the sonic pdf taken as a reference. For the correlation to make its full sense, the distributions compared are slightly adjusted so that their means correspond. This analysis allowed the comparison of the reference sonic pdf with the lidar pdf and with the average spectrum obtained from the different scaling methods presented in Sect. 3.2. In total, 46 different 10 min periods and thus, 46 different average spectra and pdfs were used for the results presented in Fig. 4. For the sake of clarity, only 


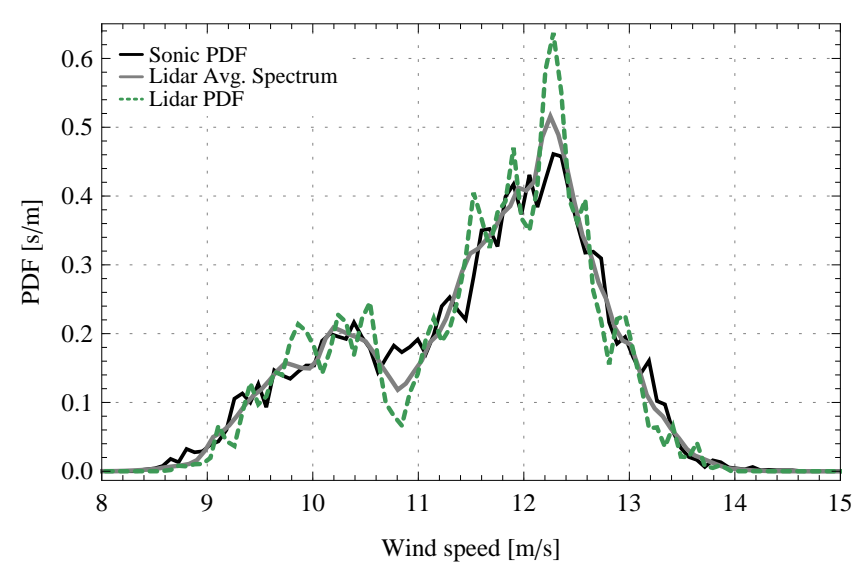

Fig. 3. Similar to Fig. 1, but for another $30 \mathrm{~min}$ period. Sonic velocities have been multiplied with 0.993 in order to compensate for the imperfect calibration shown in Fig. 2. The pdf from the lidar time series (Lidar PDF) has been added to the figure.

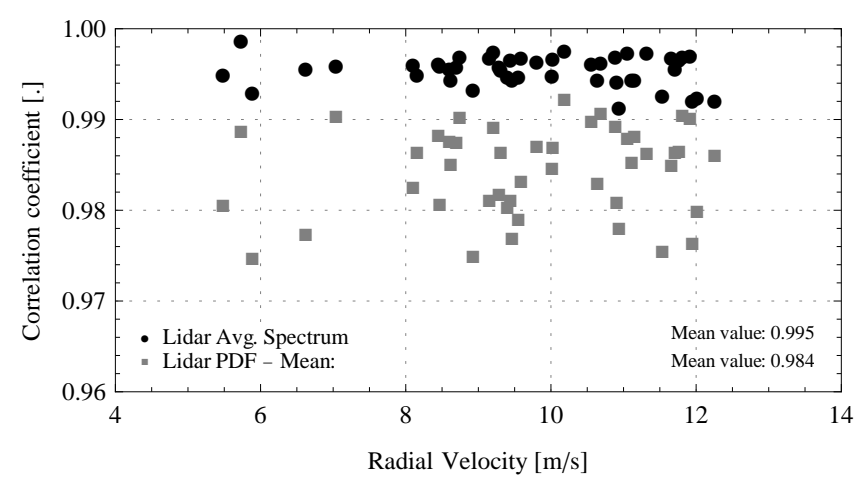

Fig. 4. Comparison of the lidar pdf and the lidar average spectrum with the sonic pdf by a correlation analysis. The radial velocity is the LOS velocity averaged over $10 \mathrm{~min}$.

the results for the method of scaling per area are shown. The correlation from the lidar wind speed time series pdf showed the lowest correlation with an average coefficient of $98.4 \%$, whereas the average spectra showed an average of $99.5 \%$. Among the different scaling methods, the method of scaling by area exhibited the best correlation results with a correlation coefficient $0.1 \%$ higher than the next best method.

A common method used for comparison of probability distributions is the Kolgomorov-Smirnov(KS) test which is based on the maximum distance between two cumulative distribution functions(cdf). The correction on the mean is applied since it is not an inherent feature of the pdf but considered as a measurement error. Results are shown in Fig. 5 using averaging periods of $30 \mathrm{~min}$. Consistent with the correlation analysis, the KS test reveals that the distance between the sonic cdf and the average spectrum cdf is in average smaller than the distance between the sonic cdf and the lidar time series cdf.

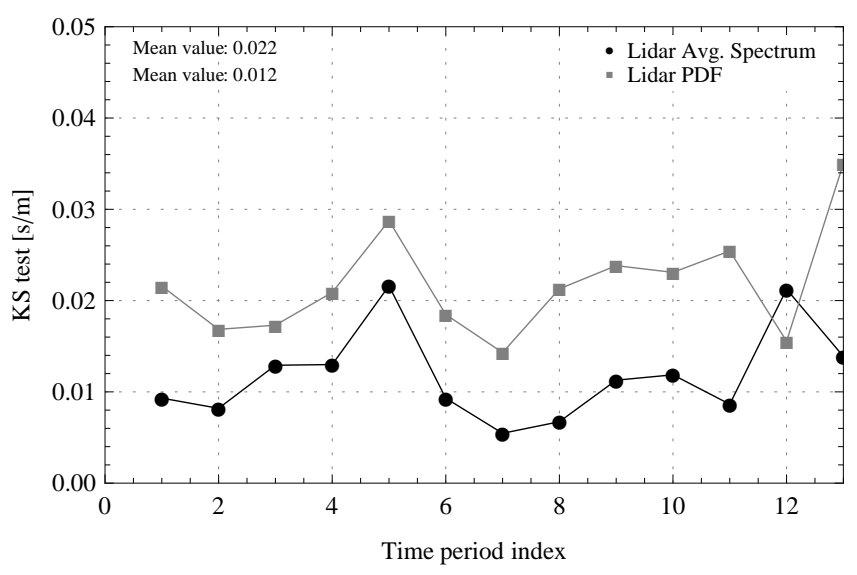

Fig. 5. Kolgomorov-Smirmov test for the two lidar statistical distributions with respect to the sonic cdf using 1430 min periods.

These results confirm what was seen graphically, i.e. the average Doppler spectrum and the sonic pdf correlates to a high degree, which in turn confirms that the assumption of a constant probability density function along the horizontal LOS is fair. Further, this comparison showed that the average Doppler spectra showed closer resemblance to the sonic pdfs than the lidar pdfs did. The potential of the method in circumventing the effect of the spatial averaging will be further emphasised by the results of the wind tunnel experiment.

\subsection{Standard deviation}

The interesting aspect of the analysis of the Doppler spectra under the assumption of a constant probability density function of the radial velocities along the LOS lies in the fact that the spatial averaging is removed. In this case, the spatial averaging inherent to the physics of the lidar disappears when the lidar spectra are averaged, whereas it is present when the lidar wind speed time series is used. This issue is critical for the determination of turbulence statistics. It has been observed and derived that the standard deviation measured by the lidar has an inherent systematic error due to this spatial averaging (Mann et al., 2009; Sjöholm et al., 2009; Angelou et al., 2012b). To illustrate the benefit of the present method, the wind speed standard deviation obtained from the lidar time series is compared to the standard deviation calculated using Eq. (17) on the average Doppler spectra. The standard deviations obtained are expressed with respect to the standard deviation measured by the sonic anemometer as shown in Fig. 6.

For this figure, all the $4610 \mathrm{~min}$ periods available were used. It is seen that the standard deviation from the average spectrum systematically shows a better agreement with the sonic standard deviation. Though this gain is minor in the field experiment, it will appear obvious from investigation of the wind tunnel experiment that a better determination of the turbulence statistics is obtained by this method. 


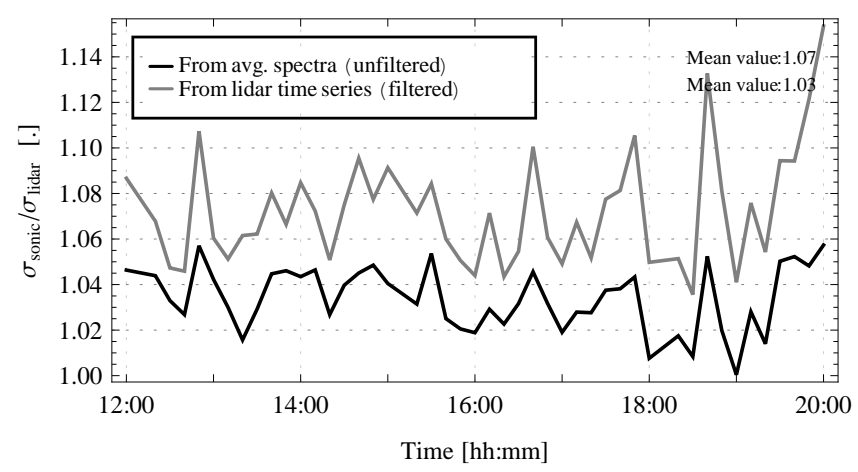

Fig. 6. Comparison of standard deviations obtained from the lidar time series (gray) and from the average Doppler spectrum (black) with respect to the sonic standard deviation.

\subsection{Wind tunnel turbulence}

We now present the results from a similar comparison in the wind tunnel.

Figure 7a shows the resulting pdfs based on the HW (blue) and lidar (green and red) measurements as described above. All three measurements find essentially the same mean wind speed of approximately $44.3 \mathrm{~m} \mathrm{~s}^{-1}$ (see Table 1), but clearly the width of the pdf based on wind speeds from velocity determination from individual Doppler spectra (red) stands out from the other two. This pdf is considerably narrower than the other two with a standard deviation of $0.30 \mathrm{~m} \mathrm{~s}^{-1}$ as compared to $0.56 \mathrm{~m} \mathrm{~s}^{-1}$. This is due to the spatial averaging inherent to the lidar as described above. The agreement between the HW pdf and the lidar mean spectrum is remarkable. This can be quantified for instance by analysis of their widths which are 0.5647 and $0.5634 \mathrm{~m} \mathrm{~s}^{-1}$, respectively.

The same tendency is clearly seen in Fig. $7 \mathrm{~b}$ showing the results of the second test series where the tunnel was set to deliver a mean speed of $55 \mathrm{~m} \mathrm{~s}^{-1}$. Again, the widths of the HW pdf and the lidar average spectrum are found to be in high correlation with standard deviations of 0.6729 and $0.6747 \mathrm{~m} \mathrm{~s}^{-1}$, respectively, while the lidar pdf is considerably narrower with a width of $0.3206 \mathrm{~m} \mathrm{~s}^{-1}$. However, it is also seen that the mean wind speeds estimated by the HW and the lidar deviate by approximately $0.18 \mathrm{~m} \mathrm{~s}^{-1}$ from each other or by approximately $0.3 \%$.

Table 1 summarises the results for all turbulence runs in the wind tunnel. The wind tunnel is equipped with a heat exchanger, which, as a side effect, removes aerosols. Therefore, a tiny quantity of smoke was introduced into the tunnel flow prior to the experimental runs. The sequence of runs in Table 1 thus corresponds to smaller and smaller aerosol loading. The average wind speeds derived from the lidar average Doppler spectrum and the histogram of lidar velocities are, as expected, virtually identical. The difference from the mean from the hotwire varies from 0 to $0.6 \%$. The standard deviations from the average Doppler spectra are vastly superior to the ones obtained from the time series of lidar velocities. The performance of the lidar deteriorates from run 1 to run 7 most probably because the particle concentration in the wind tunnel decreases as a function of time. This progression can also be seen on the pdfs shown in Fig. 7a-d.

\subsection{Laminar wind tunnel flow}

We now discuss the very narrow Doppler spectra obtained in the non-turbulent experiments in the wind tunnel. Figure 8 shows the ensemble mean of the 5824 valid spectra recorded at the pre-set speed wind speed of $50 \mathrm{~m} \mathrm{~s}^{-1}$, which is the equivalent to $45.3 \mathrm{~m} \mathrm{~s}^{-1}$ perpendicular to the beam. The Gaussian fit used for deriving the width of the average Doppler spectrum is also shown.

The procedure for obtaining the width is now repeated for every wind speed. Fig. 9 shows the $1 / e^{2}$ width of the fitted Gaussian as a function of wind speed perpendicular to the beam together with the simple theoretical prediction given in Eq. (15) and the prediction based on Eq. (13). The distributions are seen to be slightly wider than those predicted by Eq. (13). A possible explanation for this is that the flow in the wind tunnel may not be completely laminar, which could add to the width. According to Fischer (2012), the turbulence level in the "laminar" wind tunnel is very difficult to measure, but it seems that it is less than $0.2 \%$. For a mean wind speed of $50 \mathrm{~m} \mathrm{~s}^{-1}$, it would correspond to a maximum standard deviation of $0.1 \mathrm{~m} \mathrm{~s}^{-1}$. This corresponds to a frequency shift of $132 \mathrm{kHz}$, which is of the same order as the difference between the theoretical and the measured values. Therefore, by comparing the measurements and the theoretical curve of Fig. 9, we cannot rule out the possibility that weak velocity fluctuations in the wind tunnel account for the additional spectral width.

\section{Discussion}

The cw lidars used in these investigations performed at least as well as other similar investigations with respect to the mean wind speed (Wagner et al., 2011; Gottschall et al., 2012). The differences in the mean measured by the sonic are generally less than $1 \%$, and around $0.1 \%$ for the hot-wire in the wind tunnel. These small differences are of no concern here. It is the ability of the cw lidar to measure wind fluctuations by the use of the average Doppler spectrum, which is the central issue of this paper.

The graphical comparisons of the sonic pdf and the average Doppler lidar spectrum showed a strong agreement between the two curves; this agreement was also confirmed by the correlation analysis. The comparison in the wind tunnel between the pdf from the hotwire and the average Doppler spectrum from the cw lidar was equally encouraging. In both experiments, the agreement with the average Doppler spectrum was significantly better than the pdf based on lidar 
Table 1. Mean wind speeds and widths for the different instruments and analysis methods. Dimensional variables are in metres per second.

\begin{tabular}{lcccccccc}
\hline \multirow{2}{*}{ Run } & $v_{\text {set }}$ & \multicolumn{9}{c}{$\langle v\rangle$} & & \multicolumn{3}{c}{$\sigma$} \\
\cline { 9 - 10 } \cline { 8 - 9 } & & HW & Lidar avg. & Lidar PDF & & HW & Lidar avg. & Lidar PDF \\
\hline & & & & & & & \\
1 & 45 & 44.2249 & 44.2765 & 44.2668 & & 0.5647 & 0.5634 & 0.3000 \\
2 & 55 & 53.9259 & 54.1091 & 54.1015 & & 0.6729 & 0.6747 & 0.3206 \\
3 & 45 & 44.2914 & 44.2914 & 44.2749 & & 0.5539 & 0.5329 & 0.2257 \\
7 & 45 & 44.0622 & 44.3158 & 44.2610 & & 0.5626 & 0.5171 & 0.2905 \\
\hline
\end{tabular}

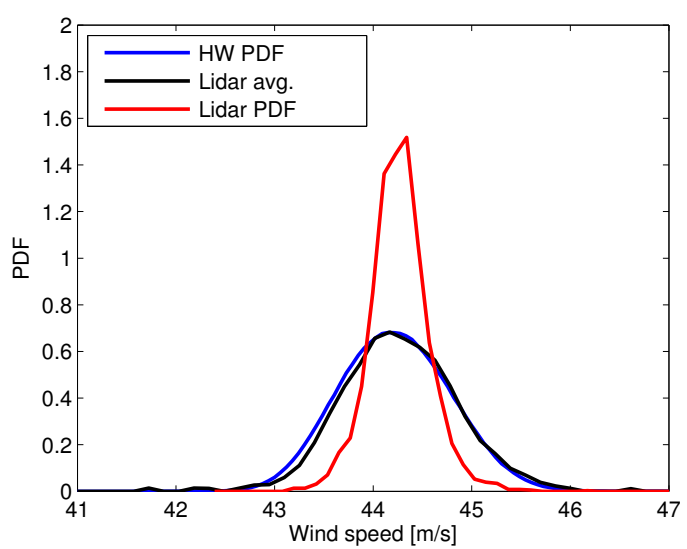

(a)

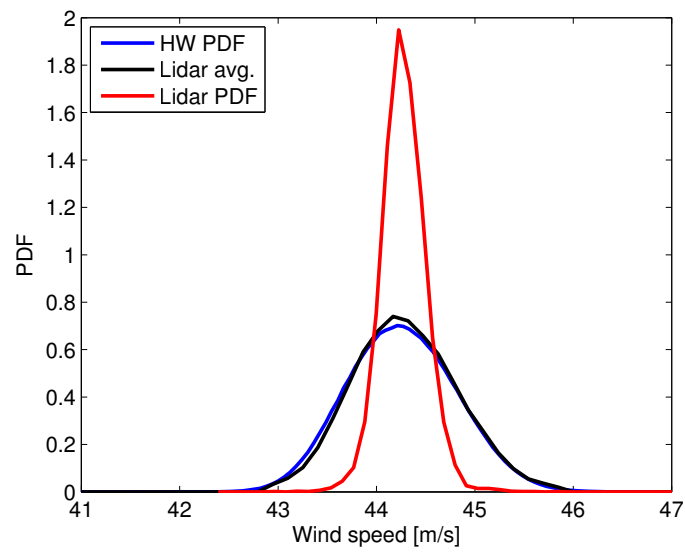

(c)

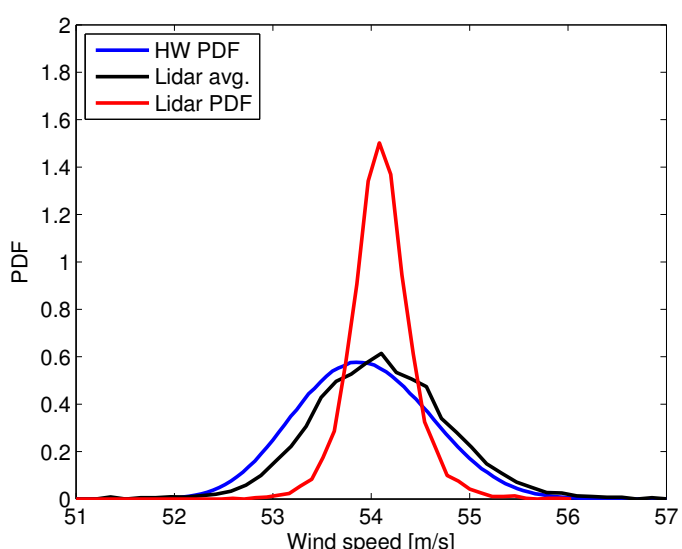

(b)

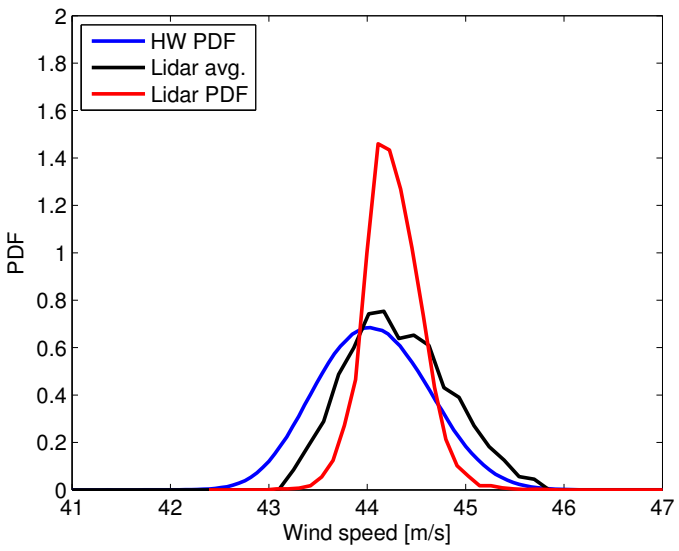

(d)

Fig. 7. The pdfs based on the HW and lidar measurements together with the ensemble averaged lidar spectrum and the pdf obtained from the lidar wind speed time series. The four figures (a-d) correspond respectively to run 1, 2, 3 and 7 . See Table 1.

derived velocities, see for example Fig. 7a, implying that the spatial averaging could be circumvented. Yet differences in standard deviations of the order of $3 \%$ are found between results from the averaging spectra method and the sonic measurements. Several factors could influence the method and explain these differences, mainly: the assumptions leading to Eq. (3), the noise suppression method and the scaling method of the spectra. In the wind tunnel experiment, the difference between the methods of obtaining turbulence statistics from the lidar is very pronounced because in this case, the scale of the turbulence is of the order of the full-width half-maximum length of the cw lidar. In the atmosphere at the focus distance used in this experiment $(67.5 \mathrm{~m})$, the measurement length is smaller than the turbulence length scale; therefore, the difference between the methods is small. However, it should be kept in mind that the measurement length increases quadratically with an increase in focus distance (Smith et al., 2006); hence, the benefit of the method will be more significant at relatively long focus distances. For the current experiment this distance was constrained by the location of the wind turbine and the meteorological mast. 


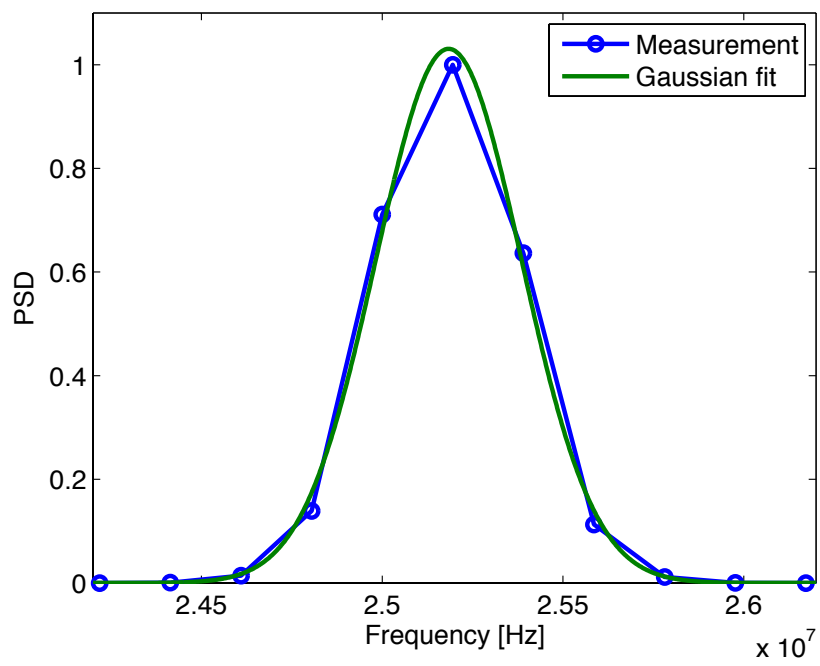

Fig. 8. Example of ensemble averaged Doppler spectrum together with a Gaussian fit obtained at a nominal wind speed of $50 \mathrm{~ms}^{-1}$.

We have also shown that neither the transit time nor the finite time period of the Fourier analysis significantly widens the average Doppler spectrum. For wind speeds perpendicular to the laser beams of $70 \mathrm{~m} \mathrm{~s}^{-1}$, spectral widths of up to $1 \mathrm{MHz}$ were observed, but that relatively large width was obtained for an extremely small beam radius and in a very homogeneous flow. For very low wind speeds, the spectral width is dominated by the rectangular window function imposed by the sampling of the signal. This width is less than two frequency bins in the digitised spectrum and is thus considerably smaller than what is usually seen in the measurements of atmospheric wind speeds. Though present for the laminar case of the wind tunnel experiment, the effect of FFT-windowing is not significant for the general case of turbulence in the atmosphere. For a conically scanning $\mathrm{cw}$ lidar, wind speeds perpendicular to the beam can easily exceed the maximum speed tested here because of the normally long focus ranges and resulting circumference of the scanned disc. However, a relatively long focus range will also lead to a relatively large beam waist radius. For example, for a ZephIR lidar focused at $100 \mathrm{~m}$ and with a scan rate of $1 \mathrm{~s}$, the tangential scan speed will be approximately $160 \mathrm{~ms}^{-1}$, but the waist radius of approximately $2 \mathrm{~mm}$ and the spectral bandwidth will be dominated by the window function. Notice also that because the tangential speed and the waist radius both increase linearly with focus distance, this is true for all ranges. Therefore, for spectral broadening to severely affect the recorded Doppler spectrum substantially higher scan rates are required.

For the method of average Doppler spectrum to be relevant, it must be applicable in situations where the flow changes statistically along the laser beam. This was shown in Mann et al. (2010) for some statistics including the covariance between the horizontal and vertical velocity com-

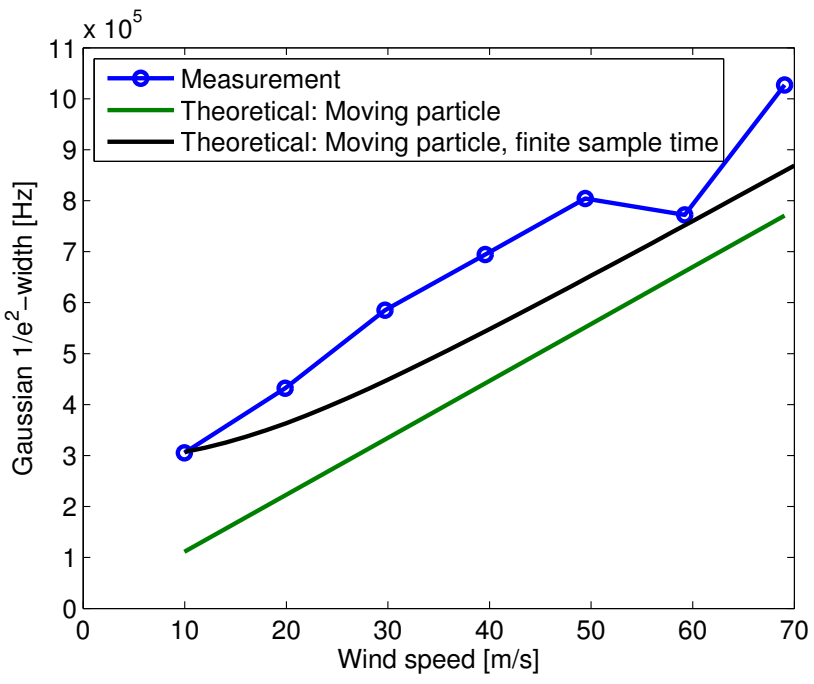

Fig. 9. Spectral bandwidth as function of wind speed. The measured values are represented by the dots, while the green and black curves indicate the theoretical values.

ponents, but remains to be verified for other important statistical quantities such as various variances relevant for siting of wind turbines in natural terrain. The first attempt of proceeding to this direction is presented in Sathe and Mann (2012b).

\section{Conclusions}

The advantage of using average Doppler spectra for the study of wind statistics was confirmed by field and wind tunnel experiments. The hypothesis that the lidar average Doppler spectrum is related to the probability density function of the radial velocities in the vicinity of the focus point was confirmed as well. This expected relation was an identity if the flow was homogeneous along the LOS, which was the case for both the field and the wind tunnel experiments.

The benefit of using the method of average Doppler spectrum for the determination of standard deviation was proved as it significantly reduced the differences in the standard deviation measured with ordinary anemometers by removing the spatial averaging effect.

Acknowledgements. The Danish National Advanced Technology Foundation's project Integration of wind liars in wind turbines for improved productivity and control (HTF 049-2009-3), Center for Computational Wind Turbine Aerodynamics and Atmospheric Turbulence funded by the Danish Council for Strategic Research grant 09-067216, and the windscanner.dk project funded by Danish Agency for Science, Technology and Innovation through grant no. 2136-08- 0022 are all gratefully acknowledged for supporting this work. The authors would like to thank the referees for their valuable and constructive comments.

Edited by: D. Feist 


\section{References}

Angelou, N., Foroughi Abari, F., Mann, J., Mikkelsen, T., and Sjöholm, M.: Challenges in noise removal from Doppler spectra acquired by a continuous-wave lidar, in: 26th International Laser Radar Conference, Greece, S5P-01, 2012a.

Angelou, N., Mann, J., Sjöholm, M., and Courtney, M.: Direct measurement of the spectral transfer function of a laser based anemometer, Rev. Sci. Instrum., 83, 033111, doi:10.1063/1.3697728, 2012b.

Berg, J., Mann, J., Bechmann, A., Courtney, M. S., and Jørgensen, H. E.: The Bolund Experiment, Part I: Flow over a steep, three-dimensional hill, Bound.-Lay. Meteorol., 141, 219243, doi:10.1007/s10546-011-9636-y, 2011.

Cariou, J.-P., Augere, B., and Valla, M.: Laser source requirements for coherent lidars based on fiber technology. C. R. Phys., 7, 213223, doi:10.1016/j.crhy.2006.03.012, 2006

Drobinski, P., Dabas, A., and Flamant, P.: Remote measurement of turbulent wind spectra by heterodyne Doppler lidar technique, J. Appl. Meteorol., 39, 2434-2451, 2000.

Emeis, S., Harris, M., and Banta, R. M.: Boundary-layer anemometry by optical remote sensing for wind energy applications, Meteorol. Z., 16, 337-347, doi:10.1127/0941-2948/2007/0225, 2007.

Fischer, A.: Hot Wire Anemometer Turbulence Measurements in the wind Tunnel of LM Wind Power, DTU Wind Energy report E-0006, DTU Wind Energy, Roskilde, Denmark, 2012.

Frehlich, R. and Cornman, L.: Estimating spatial velocity statistics with coherent Doppler lidar, J. Atmos. Ocean. Tech., 19, 355366, 2002.

Gottschall, J., Courtney, M. S., Wagner, R., Jørgensen, H. E., and Antoniou, I.: Lidar profilers in the context of wind energy-a verification procedure for traceable measurements, Wind Energy, 15, 147-159, doi:10.1002/we.518, 2012.

Kristensen, L., Kirkegaard, P., and Mikkelsen, T.: Determining the Velocity Fine Structure by a Laser Anemometer with Fixed Orientation, Tech. Rep. Risø-R-1762(EN), Risø DTU, 2011.

Lothon, M., Lenschow, D. H., and Mayor, S. D.: Doppler lidar measurements of vertical velocity spectra in the convective planetary boundary layer, Bound.-Lay. Meteorol., 132, 205-226, 2009.

Mann, J., Cariou, J.-P., Courtney, M. S., Parmentier, R., Mikkelsen, T., Wagner, R., Lindelöw, P., Sjöholm, M., and Enevoldsen, K.: Comparison of 3D turbulence measurements using three staring wind lidars and a sonic anemometer, Meteorol. Z., 18, 135-140, 2009.

Mann, J., Peña, A., Bingöl, F., Wagner, R., and Courtney, M. S.: Lidar scanning of momentum flux in and above the surface layer, J. Atmos. Ocean. Technol., 27, 959-976, doi:10.1175/2010JTECHA1389.1, 2010.
O'Connor, E. J., Illingworth, A. J., Brooks, I. M., Westbrook, C. D., Hogan, R. J., Davies, F., and Brooks, B. J.: A method for estimating the turbulent kinetic energy dissipation rate from a vertically pointing Doppler lidar, and independent evaluation from balloon-borne in situ measurements, J. Atmos. Ocean. Technol., 27, 1652-1664, 2010.

Pedersen, A., Montes, B., Pedersen, J., Harris, M., and Mikkelsen, T.: Demonstration of short-range wind lidar in a high-performance wind tunnel, in: Proceedings of EWEA 2012, EWEA - The European Wind Energy Association, Copenhagen, Denmark, 2012.

Pichugina, Y. L., Banta, R. M., Kelley, N. D., Jonkman, B. J., Tucker, S. C., Newsom, R. K., and Brewer, W. A.: Horizontalvelocity and variance measurements in the stable boundary layer using Doppler lidar: sensitivity to averaging procedures, J. Atmos. Ocean. Technol., 25, 1307-1327, 2008.

Sathe, A. and Mann, J.: Measurement of turbulence spectra using scanning pulsed wind lidars, J. Geophys. Res.-Atmos., 117, D01201, doi:10.1029/2011JD016786, 2012a.

Sathe, A. and Mann, J.: Turbulence measurements using six lidar beams, in: Extended Abstracts of Presentations from the 16th International Symposium for the Advancement of Boundary-Layer Remote Sensing, Boulder, Colorado, USA, 302-305, 2012 b.

Sathe, A., Mann, J., Gottschall, J., and Courtney, M. S.: Can wind lidars measure turbulence?, J. Atmos. Ocean. Technol., 28, 853868, doi:10.1175/JTECH-D-10-05004.1, 2011.

Sjöholm, M., Mikkelsen, T., Mann, J., Enevoldsen, K., and Courtney, M.: Time series analysis of continuous-wave coherent Doppler lidar wind measurements, Meteorol. Z., 18, 281-287, 2009.

Smith, D. A., Harris, M., Coffey, A. S., Mikkelsen, T., Jørgensen, H. E., Mann, J., and Danielian, R.: Wind lidar evaluation at the Danish wind test site Høvsøre, Wind Energy, 9, 87-93, 2006.

Sonnenschein, C. M. and Horrigan, F. A.: Signal-to-noise relationships for coaxial systems that heterodyne backscatter from the atmosphere, Appl. Optics, 10, 1600, doi:10.1364/AO.10.001600, 1971.

Spiegelberg, C., Geng, J., Hu, Y., Kaneda, Y., Jiang, S., and Peyghambarian, N.: Low-Noise Narrow-Linewidth Fiber Laser at $1550 \mathrm{~nm}$, J. Lightwave Technol., 22, 57-62, 2004

Wagner, R., Courtney, M., Gottschall, J., and LindelöwMarsden, P.: Accounting for the speed shear in wind turbine power performance measurement, Wind Energy, 14, 993-1004, doi:10.1002/we.509, 2011. 